\title{
Happenings étudiants et théâtre de rue : subversion de l'espace public autour de 1968
}

Studentische Happenings und Straßentheater: subversives Umfunktionieren des öffentlichen Raums um 1968

Student Happenings and Street Theatre: Subversion of Public Space In and Around 1968

\section{Charlotte Bomy}

\section{OpenEdition}

Journals

Édition électronique

URL : https://journals.openedition.org/ceg/7845

DOI : $10.4000 /$ ceg.7845

ISSN : 2605-8359

\section{Éditeur}

Presses Universitaires de Provence

Édition imprimée

Date de publication : 1 mai 2013

Pagination : 53-68

ISBN : 0751-4239

ISSN : 0751-4239

\section{Référence électronique}

Charlotte Bomy, « Happenings étudiants et théâtre de rue : subversion de l'espace public autour de

1968 », Cahiers d'Études Germaniques [En ligne], 64 | 2013, mis en ligne le 02 janvier 2020, consulté le

02 juin 2021. URL : http://journals.openedition.org/ceg/7845 ; DOI : https://doi.org/10.4000/ceg.7845 


\section{Happenings étudiants et théâtre de rue : subversion de l'espace public autour de 1968}

Charlotte BOMY

Université Montpellier 3/CREG EA 4151

En liaison avec les mouvements contestataires de la fin des années 1960, les arts du spectacle font irruption dans l'espace public, avec une volonté de renouvellement portée par la conjoncture culturelle de l'époque qui cherche à abolir les frontières entre l'art, la politique et la vie. À Berlin-Ouest, comme dans d'autres villes allemandes, le théâtre investit la rue et la rue investit le théâtre pour s'adresser à un nouveau public, pour créer des événements à dimension festive et militante. Quels types de formes spectaculaires voit-on émerger à cette époque dans le sillage de la contestation étudiante et quel rapport l'institution théâtrale entretient-elle avec les acteurs de ces productions marginales ? Dans quelle mesure est-il possible de délimiter un espace culturel de contestation ou un "espace contre-culturel théâtral » spécifique à Berlin-Ouest ?

Grâce à une contextualisation de phénomènes parfois associés au happening, nous proposons d'abord de présenter la manière dont ses nouvelles formes spectaculaires s'articulent sur le mode de théâtralité politique induit par le mouvement étudiant. Dans cette période de révolte globale contre le système, l'institution théâtrale est également interpellée par des personnalités issues du monde théâtral qui tentent de renouveler, à l'intérieur de ses murs, ses modes de fonctionnement et ses formes artistiques. L'exemple de Peter Handke, à l'époque observateur critique de tous ces événements à Berlin, permet de comprendre la pertinence de ces nouvelles impulsions protestataires pour la recherche artistique en étudiant la façon dont celles-ci sont finalement intégrées à un discours théâtral plus légitime. Nous aborderons dans un dernier temps les répercussions liées à l'émergence d'un théâtre de rue amateur sur l'activité théâtrale professionnelle, avec en particulier, dans les années 1970 , la naissance et la structuration d'une scène off qui se développera par la suite sous le nom de Freies Theater (« théâtre libre $\gg$ ). 
Le propos de cet article est illustré par l'analyse de photographies d'archive qui permettent de reconstruire en partie l'objet d'étude si particulier que constitue le spectacle vivant: il s'agit d'un objet qui n'est pas fixé dans le temps comme une partition ou un livre (voire un texte de théâtre ou une scénographie reproductibles) et qui contient de multiples dimensions et informations : Barthes parlait à ce sujet de la "polyphonie $»^{1}$ signifiante $\mathrm{du}$ théâtre. Soulignons que la démarche n'est pas facilitée lorsqu'on travaille sur des productions marginales, développées hors de l'institution (qui, elle, archive et filme le travail artistique), ou encore sur des groupes qui, au nom du travail politique, ont souvent renoncé au succès public ou critique, et dont les archives sont quasi-inexistantes.

\section{Contestation et théâtralisation du politique à Berlin}

Sans remonter à la tradition du théâtre de bateleurs sur la place publique qui se proposait de divertir le (bas) peuple par des bouffonneries ou des acrobaties d'un art scénique extra muros plus gestuel que textuel, la performativité politique de l'agit-prop weimarienne au sein du mouvement ouvrier dans les années 1920 montrait déjà que ce phénomène prenait toutefois une dimension contre-culturelle véritable lorsqu'il était adossé à un vaste mouvement socio-historique contestataire comme celui de la fin des années 1960. Pour circonscrire ces événements, commençons par quelques remarques historiques sur la spécificité des mouvements de contestation sur le territoire de la République Fédérale d'Allemagne, et à Berlin-Ouest en particulier. On peut dire qu'il s'agit d'un processus résultant d'un assemblage composite et hétérogène, qui, dans les années 1966-1968, finit par agréger plusieurs mouvements sous le terme d'opposition extra-parlementaire (APO). C'est, d'une part, le mouvement des marches de Pâques (Ostermarschbewegung) pour le désarmement nucléaire ${ }^{2}$; le mouvement d'opposition aux lois d'urgence (Notstandsgesetze) fédère, quant à lui, les syndicalistes, les intellectuels et des étudiants - pour la défense des droits fondamentaux et dans la mesure où ces projets législatifs constituent une menace pour le droit de grève ${ }^{3}$. Enfin, le mouvement contestataire étudiant, lui-même très

1 Roland BARTHES, « Littérature et signification », in Essais critiques, Paris, Seuil, 1981, p. 258.

2 En 1960, à Hambourg, la première marche pour le désarmement nucléaire marque la naissance du mouvement des Marcheurs de Pâques, dont la capacité de mobilisation s'accrut ensuite régulièrement. Ce mouvement s'inspire du modèle de la campagne pour le désarmement nucléaire lancé en Grande-Bretagne à la fin des années 1950 par Bertrand Russel, tout en se situant à la marge de la tradition des luttes du mouvement ouvrier.

3 Le projet des lois d'urgence entendait combler un vide juridique dans la souveraineté encore incomplète de la RFA depuis la conclusion des accords des Alliés occidentaux en 1954 En janvier 1960, un texte de loi est proposé par le Ministère fédéral de l'Intérieur, il s'inspire de l'article 48 de la constitution de Weimar, qui avait permis l'instauration de la dictature présidentielle lors de la crise de la République de Weimar, dans la tradition de l'État autoritaire 
diversifié, s'organise durant ces années sur fond de circulations et de transferts transnationaux : il se dresse d'abord contre la domination autoritaire sous toutes ses formes (dans la société et dans l'État), en revendiquant plus de démocratie, de transparence et de participation démocratique. À l'intérieur de l'APO, c'est le moment de l'essor du $\operatorname{SDS}^{4}$ qui va instituer des cercles de travail dans les universités en commentant de manière non-conformiste les classiques du marxisme, et en s'interrogeant notamment sur les nouvelles modalités de l'articulation entre théorie et pratique - dans l'esprit de la « nouvelle gauche » américaine. À propos du mouvement étudiant, soulignons une spécificité propre à la RFA par rapport au reste de l'Europe : la dynamique protestataire est également le produit d'un conflit de générations en relation avec l'expérience de la Seconde Guerre mondiale et la critique du passé nazi que les très médiatisés "Procès d'Auschwitz » (196365 ) avaient mis à l'ordre du jour. Le besoin de révolte et de démocratisation se fait particulièrement ressentir chez une génération sensibilisée, à partir du début des années 1960, au thème de la continuité des élites du III $^{\mathrm{e}}$ Reich à la RFA.

Berlin-Ouest joue, après des préludes à Francfort sur le Main, un rôle essentiel dans l'impulsion de la contestation. Son statut est particulier en Allemagne : il s'agit de la seule ville de RFA où le service militaire n'est pas obligatoire, puisqu'elle a le statut de «ville démilitarisée » depuis la Seconde Guerre mondiale. Elle représente un pôle d'attraction pour la jeunesse alternative d'Allemagne de l'Ouest, une population qui non seulement va former le noyau dur de la révolte étudiante, mais également façonner la mentalité de la ville dans les années 1960 et 1970, en favorisant la naissance d'une culture alternative, le plus souvent appelée en allemand "deuxième culture ». La contestation anti-autoritaire débute de fait plus tôt à Berlin que dans le reste de l'Allemagne, et même de l'Europe. Dès le semestre d'hiver 1965/1966 - que l'on a appelé par la suite le «semestre du Vietnam », le SDS était parvenu à orchestrer une série d'actions, de slogans et de manifestations dont Rudi Dutschke était le principal leader. Au cours de cette période, les contestataires expérimentent de nouvelles techniques de manifestation, empruntées au mouvement des étudiants américains : du go-in (intervention collective lors d'un cours ou d'une conférence) au sit-in (manifestation assise et immobile visant à occuper un espace public), en passant par le teach-in

allemand (Obrigkeitsstaat). La proposition se heurte à l'opposition conjuguée des syndicats, de la SPD et des Länder. Voir : Justine FAURE et Denis ROLLAND (dir.), 1968 hors de France. Histoire et constructions historiographiques, Paris, L'Harmattan, coll. « Inter-National », 2009.

4 En tant qu'organisation étudiante, le SDS ou Sozialistischer Deutscher Studentenbund avait pris ses distances aussi bien avec les positions pragmatiques du parti social-démocrate (SPD), qu'avec les positions orthodoxes du parti communiste allemand (KPD). À la suite de nombreux désaccords internes, le SDS est finalement dissous le 11 mars 1970. 
(discussion ouverte et sans contrainte de temps dont le but est l'action politique concrète) $)^{5}$.

Ces formes de manifestation et de prises de parole caractérisées par une forte visibilité dans l'espace public, vont s'amplifier à partir du 2 juin 1967, date de la visite officielle du couple impérial iranien à Berlin-Ouest. Le voyage du shah nourrit la controverse et provoque une grande manifestation : on dénonce la réalité d'un régime oppressif à l'égard des ouvriers, des paysans et des étudiants, qui plus est dans un pays devenu un pion stratégique de l'impérialisme américain. Le scandale provoqué par la mort de l'étudiant Benno Ohnesorg (tué par le policier Karl-Heinz Kurras) lors de cette manifestation va constituer le point de départ d'une intensification et d'une radicalisation de la mobilisation étudiante qui durera jusqu'à l'automne 1968. Cet événement élargit les soutiens des contestataires : une grande partie du corps professoral de la Freie Universität Berlin rejoint le camp des étudiants contre les méthodes de la presse, en particulier du groupe d'Axel Springer, éditeur de la Bild-Zeitung.

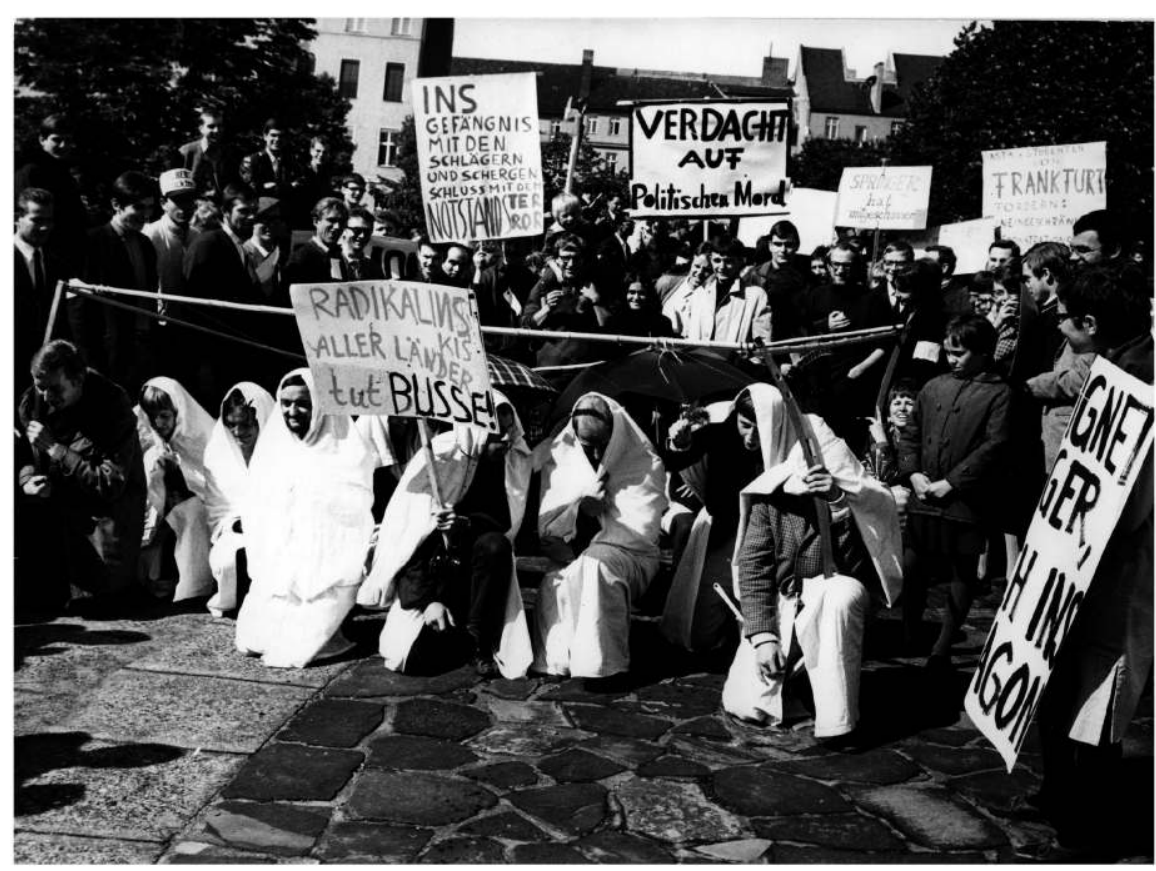

Ill. 1 : La marche des pénitents : manifestation d'étudiants ouest-berlinois quelques jours après l'assassinat de Benno Ohnesorg, 13.06.1967. PressebildVerlag Schirner, (C) Deutsches Historisches Museum, Berlin.

5 Voir à ce sujet l'ouvrage de Dorothea KRAUSE, Theater-Proteste. Zur Politisierung von Theater und Bühne in den 1960er Jahren, Frankfurt/Main, Campus Verlag, 2007. La troisième partie du livre concerne plus spécifiquement les stratégies de mobilisation étudiantes. 
On peut dire de cette génération révoltée qu'elle est consciente de la manipulation de l'opinion publique par les médias de masse ; elle fait ellemême les frais des mises en scènes médiatiques orchestrées par la BildZeitung, qui dramatise et dépolitise les actions étudiantes en les vidant de leur contenu, pour n'en conserver que l'aspect violent ou sensationnel. C'est d'une certaine manière pour répondre aux moyens de communication généralisés - à une époque où la télévision devient un média dominant - que les étudiants contestataires essaient de déjouer l'image que donnent d'eux les mass media, en s'appropriant et en (re)théâtralisant l'espace public, en faisant de la contestation un spectacle mobile et ludique dans la tradition de l'agitprop weimarienne que le mouvement va contribuer à redécouvrir. La « marche des pénitents » qui a lieu le 13 juin 1967, une dizaine de jours après l'onde de choc provoquée par la mort de Benno Ohnesorg, est un exemple de manifestation alternative déployant un nouveau type de visibilite (ill. 1). Ce happening est une réaction à la décision du maire de Berlin, Heinrich Albertz, qui avait d'abord tenté d'interdire toute manifestation. Il se dresse également contre la police berlinoise qui cherche alors à réguler les mouvements contestataires en exigeant des organisateurs qu'ils inscrivent leurs noms et prénoms sur un bandeau autour du bras, afin de pouvoir mieux les " ficher ». Pour manifester leur mécontentement face à la surveillance policière, les étudiants du SDS choisissent la provocation par le « surjeu» et défilent à genoux, drapés de blanc, avec des pancartes portant des inscriptions telles que «Extrémistes de tous les pays, faites à présent pénitence ».

La contestation anti-autoritaire, qui donne le ton avec des actions plus ou moins spectaculaires à Berlin-Ouest, est aussi le fait de personnalités anarchistes, non conventionnelles et intellectuelles, rassemblées sous le toit de la Kommune 1. Sur fond d'activisme esthétique et politique, ses membres se mettent en scène à travers des actions directes ou happenings : 1 '« attentat au pudding $»^{6}$ en est une forme emblématique, relayée mensongèrement par la presse et menant à l'arrestation de Fritz Teufel et Rainer Langhans. Les communards sont régulièrement sanctionnés par la police qui ne reconnaît pas le caractère ludique de leurs actions, même si, le plus souvent, celles-ci prennent la forme d'une contestation pacifiste : cortèges dansants déguisés sur le Kudamm pour dénoncer le caractère irrationnel de l'ordre rationnel, ou

6 Au mois d'avril 1967, pour protester contre la visite du vice-président américain Humphrey à Berlin-Ouest, les membres de la Kommune 1 préparèrent un happening connu sous le nom «d'attentat au pudding ». La police arrêta deux d'entre eux, Fritz Teufel et Rainer Langhans, en train de préparer ce que le journal Bild-Zeitung devait qualifier « d'engin explosif », mais qui était en réalité une «bombe » faite de fumigènes, de farine et de yaourt Cette action engendra des tensions entre le SDS et la Kommune 1. Le 3 mai 1967, le SDS exclut les communards pour cause d'actionnisme anarchiste et pour " ignorance démonstrative du travail théorique ». Voir à ce sujet : Joachim SCHARLOTH, «Ritualkritik und Rituale des Protests. Die Entdeckung des Performativen in der Studentenbewegung der 1960er Jahre », in Martin KLIMKE, Joachim SCHARLOTH (dir.), 1968. Ein Handbuch zur Kultur- und Mediengeschichte der Studentenbewegung, Stuttgart/Weimar, Metzler, 2007. 
encore bulles de savon "soufflées » sous le nez de la police signifiant le caractère vain et inutile du discours des autorités.

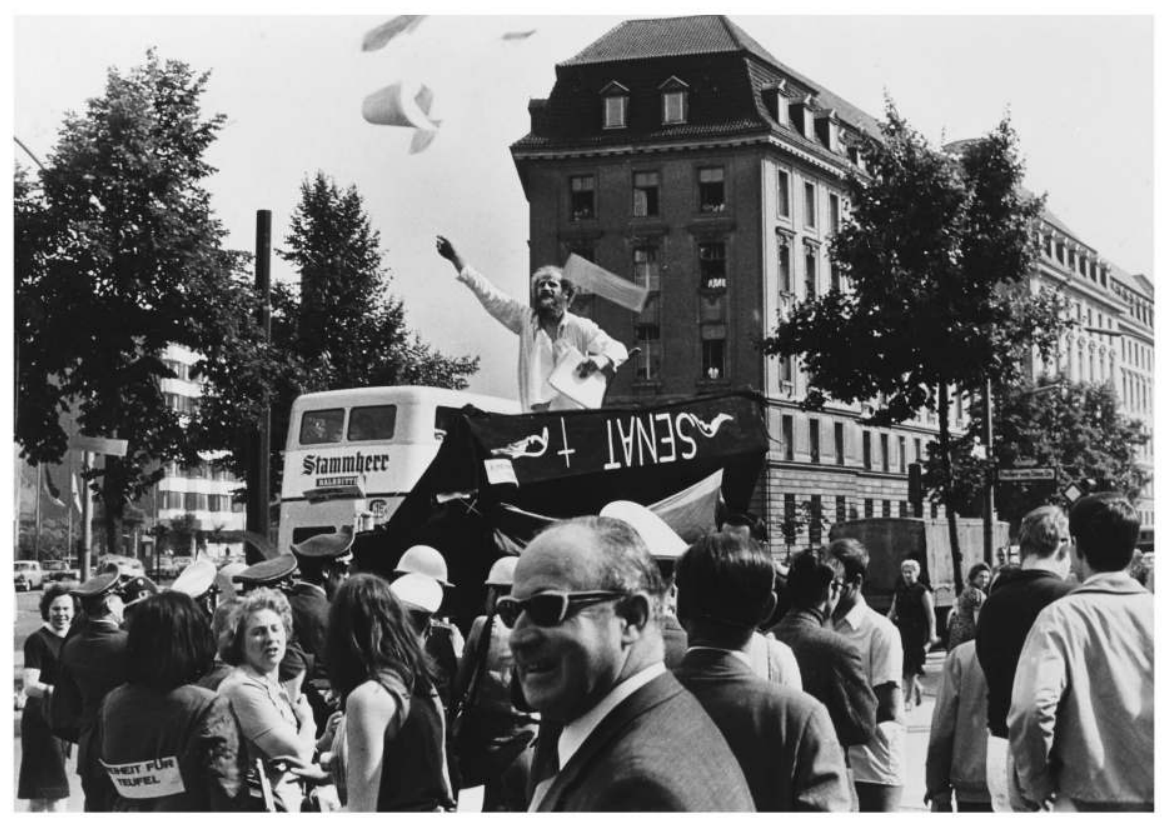

Ill. 2 : Happening et contre-enterrement de Dieter Kunzelmann devant la mairie de Schöneberg pour soutenir la libération de Fritz Teufel, 9.06.1967.

Ullstein Bild, (C) Burger.

C'est également à travers des détournements d'événements publics que les apparitions des membres de la Kommune 1 sont mises en scène. Le « happening de l'enterrement » (ill. 2), réalisé le 9 août 1967, constitue une action à la fois subversive et participative. Lors des obsèques réelles de l'ancien président du Reichstag Paul Löbe (SPD), devant la mairie de Schöneberg, Dieter Kunzelmann propose un « contre-enterrement » face à l'enterrement officiel : il sort d'un cercueil en lançant des tracts avec l'inscription «Freiheit für Teufel » («Liberté pour Teufel ») à la foule qui l'entoure. La performance visant à soutenir la libération de Fritz Teufel (alors en détention provisoire) est, comme souvent, reliée à une Flugblattaktion, c'est-à-dire une distribution intempestive de tracts. Au-delà de la provocation volontaire et de la dimension parodique de ce type de happening, il faut souligner l'impact visuel et médiatique de ces actions théâtralisées, relevant d'une véritable stratégie de communication. Alliant engagement politique, volonté de détournement et visibilité «festive ", les membres de la Kommune 1 furent à juste titre qualifiés de Medienprofis (« professionnels des médias »). 


\section{Théâtre de rue vs théâtre-théâtre}

Les mouvements révolutionnaires étudiants, à Berlin-Ouest comme dans le reste de l'Allemagne, vont secouer l'activité théâtrale professionnelle. Si l'institution théâtrale, comme toute institution, devient la cible des critiques, car elle est considérée comme apolitique, autoritaire, anti-révolutionnaire, il serait toutefois réducteur d'opposer de manière binaire une contre-culture révolutionnaire et émancipatrice à une « haute culture » (« Hochkultur ») de répertoire, établie et figée. Cette opposition est toujours à nuancer ou à préciser, l'héritage intellectuel et artistique de la première étant partiellement repris par la seconde, et inversement. On constate ainsi qu'un vent de révolte s'infiltre dans la programmation de plusieurs grandes scènes allemandes à la fin des années 1960. Dans un article publié en août 1968 dans la revue Theater heute, Henning Rischbieter passe en revue quelques productions marquantes de la saison passée : le Diskurs über Viet Nam (Discours sur le Vietnam) de Peter Weiss est créé à Francfort en 1967; la pièce Notstandsübung (Exercice d'état d'urgence) de Michael Hatry ayant pour thème le meurtre de Benno Ohnesorg est montée à Ulm, tandis qu'au Schauspielhaus de Hambourg, on peut voir Im Kongo (Une saison au Congo) de Aimé Césaire. Bien que ces productions restent des phénomènes marginaux au sein des théâtres allemands ${ }^{8}$, elles manifestent le développement d'une réflexion critique sur le rôle du théâtre dans une société en transition. De nombreuses actions, initiées par les gens de théâtre euxmêmes, viennent également ponctuer ou interrompre les représentations, en particulier lors des mouvements de protestation contre les lois d'urgence ${ }^{9}$.

Cette ouverture à l'actualité politique brûlante s'accompagne d'une autocritique et de propositions visant une forme de travail plus démocratique à l'intérieur de l'institution théâtrale. En avril 1968, les jeunes comédiens Barbara Sichtermann et Jens Johler rédigent un manifeste pour le magazine Theater heute qui dénonce «l'esprit autoritaire du théâtre allemand ${ }^{10}$. Ils invoquent un besoin de renouvellement du dialogue au sein de l'institution et revendiquent un processus de création collective où le statut du comédien serait revalorisé dans les décisions artistiques, face à celui de l'auteur ou du

7 Henning RISCHBIETER, «Die Revolte und das Theater. Ein Lagebericht aus dem Sommer $1968 »$, Theater heute (Jahrbuch), 8/1968.

8 Pour une analyse détaillée de ces mises en scène, je renvoie au dossier réalisé par Henning RISCHBIETER, « Spielformen des politischen Theaters », Theater heute, 4/1968, p. 815 .

9 Par exemple le 28 mai 1968, à la Schaubühne am Halleschen Ufer, lorsque Martin Sperr vient lire un texte incitant les comédiens allemands à faire grève, au milieu de la représentation de La Jungle des villes de Brecht, mise en scène par Peter Stein. Cette interruption devient la base d'une discussion avec le public après le spectacle. Voir à ce sujet la série d'articles regroupés dans la rubrique collective Dokumentation, sous le titre «Die demolierte Aura oder Was hält das Theater alles aus? », Theater heute, 7/1968.

10 Barbara SICHTERMANN et Jens JOHLER, « Über den autoritären Geist des deutschen Theaters "), Theater heute, 4/1968. p. 2 
metteur en scène. Il est fort probable que la tournée du Living Theater, de passage en Europe et notamment à Berlin à partir de 1966 dans le cadre des Berliner Festspiele, ait joué un rôle dans cette prise de conscience : discussion et méditation collectives faisaient partie du quotidien des membres de la troupe dirigée par Julian Beck et Judith Malina, qui est considérée comme un modèle de communauté artistique révolutionnaire et utopique pour toute une génération. Face à la difficulté d'envisager une telle forme de travail dans le réseau élaboré et hiérarchisé des Stadttheater (théâtres municipaux) et Staatstheater (théâtres d'État, financés par le Land), la seule solution semble être de constituer des troupes hors du système établi - une volonté partagée et revendiquée par de nombreux artistes qui va permettre d'infléchir la politique culturelle. À Berlin-Ouest, la refondation de la Schaubühne am Halleschen Ufer en 1970 représente à ce titre une réussite d'alternative démocratique. Animé par Peter Stein et Claus Peymann, ce « contre-modèle d'institution » revendique une gestion collective, proposant une nouvelle division du travail, à l'opposé de ce qui se pratiquait dans les autres théâtres municipaux de la ville, comme au Schillertheater ou au Schlossparktheater ${ }^{11}$.

Si la remise en question de la fonction politique du théâtre passe nécessairement par un repositionnement de l'organisation théâtrale, il faut également souligner l'impact des mouvements contre-culturels de cette époque sur la façon même de faire du théâtre. Les années autour de 1968 sont marquées par l'arrivée dans le paysage théâtral institutionnel de personnalités importantes comme Peter Zadek, Klaus-Michael Grüber, Peter Stein ou Claus Peymann. Des metteurs en scène qui affichent dès leurs débuts une volonté d'affranchissement à l'égard de la lecture académique des œuvres, ouvrant un espace d'expérimentation dans les grands théâtres de RFA. D'un point de vue historique, il est intéressant de constater que ce Regietheater ${ }^{12}$ (littéralement " théâtre de metteurs en scène ") s'est par la suite imposé comme un standard du théâtre allemand (aujourd'hui représenté par Thomas Ostermeier et fortement critiqué par un Daniel Kehlmann). On voit, là encore, de quelle manière la dynamique impulsée par la contre-culture estudiantine de la fin des années 1960 s'est finalement officialisée avec le temps, comment celle-ci a pu finalement être récupérée par la culture institutionnelle.

L'importance du happening autour de 1968 et des différents modes d'investissement de l'espace public permet toutefois d'aborder une autre voie de renouvellement pour le théâtre : celle d'un théâtre d'intervention hors de l'institution. Pour illustrer cette nouvelle opposition, on peut s'appuyer sur un article publié dans le Theater heute d'avril 1968 par Peter Handke, alors installé à Berlin-Ouest, et qui s'intitule «Straßentheater und Theatertheater ». Dans ce texte, Handke oppose le «théâtre de rue » à ce qu'il appelle le

$11 \mathrm{Au}$ sujet des fondements et de l'évolution politique et esthétique de la Schaubühne, consulter la contribution de Catherine Mazellier-Lajarrige dans ce numéro.

12 La notion de Regietheater ainsi que les metteurs en scène cités plus haut mériteraient individuellement des développements plus détaillés. Je renvoie à ce sujet à : Günther RÜHLE, Anarchie in der Regie?, Frankfurt/Main, Suhrkamp, 1982. 
" théâtre-théâtre ", c'est-à-dire le théâtre que l'on ne voit qu'au théâtre. Il revient à cette occasion sur l'importance de l'héritage brechtien dans le théâtre contemporain et tisse des liens avec les actions de la Kommune 1. Handke insiste sur la nécessité de faire de la contradiction une forme constituante de la représentation théâtrale, à la manière dont Brecht concevait le théâtre, particulièrement dans ses pièces d'apprentissage - c'est-à-dire comme une machine permettant de déployer des jeux d'apprentissage didactique $^{13}$ :

[...] Aus diesen Widersprüchen hat Brecht Spiele gemacht, Spiele: in dieser Beziehung ist die Kommune in Berlin mit Fritz Teufel als Oberhelden die einzige Nachfolgerin Brechts, sie ist ein Berliner Ensemble von einer Wirksamkeit, die jener des legitimen Berliner Ensembles entgegengesetzt ist: das legitime Ensemble errichtet Widersprüche nur, um am Schluß ihre mögliche Auflösung zu zeigen, und es zeigt Widersprüche, die in der eigenen Gesellschaftsform zumindest formal nicht mehr bestehen - kann deswegen auch zu keinem Widersprechen führen: die Kommune aber hat neu gedacht, hat ihre Widerspruchsspiele von akzeptierten Widerspruchsorten (den Theaterhäusern) auf (noch) nicht akzeptierte Widerspruchsorte verlegt, hat ihre Spiele nicht am Schluß mit einmal gemachten, schon fertigen Rezepten der Neuordnung versehen (addiert), weil die Spiele selbst, die Form des Spielens sich schon als Rezept der Neuordnung anbot. ${ }^{14}$

La Kommune 1 se présenterait donc comme le digne successeur de Brecht, mais d'un Brecht encore non consacré comme classique (comme c'est le cas en RDA où il est néanmoins rarement joué ailleurs qu'au Berliner Ensemble). C'est une sorte de Berliner Ensemble de Berlin-Ouest, non légitime, mais dont l'efficacité est redoutable. Pour Handke, le véritable Berliner Ensemble, à Berlin-Est donc, est un musée qui ne met en scène que des contradictions désuètes dans la société actuelle. La Kommune 1, en revanche, permet de repenser le théâtre de la contradiction en déplaçant l'espace de jeu du théâtre à l'extérieur de l'institution, dans la rue ${ }^{15}$. Grâce à des actions politiques théâtralisées, elle régénère par sa

13 Cf. l'entretien avec Maurice Taszman dans ce numéro.

14 Peter HANDKE, « Straßentheater und Theatertheater », Theater heute, 04/1968, p. 6-7.

15 Signalons que e'est ce que souhaite également Brecht qui, dans l'un de ses derniers textes (à l'occasion d'une rencontre avec les étudiants de théâtre de Leipzig en 1956), plaide pour un retour à l'agit-prop. Brecht propose à cette occasion le retour à de petites formes théâtrales : «wie wir, abgesehen von dem grossen Stück für die Theater, wieder zu kleinen, wendigen Kampfformen kommen [...]. »; plus loin, il évoque une forme de cohabitation entre la culture théâtrale dominante de RDA et une tradition violemment contre-culturelle weimarienne (du théâtre amateur d'agitation) à laquelle il avait participé avec Hanns Eisler et que les responsables culturels de RDA ont mise au rencart, privélégiant la « grande forme » et les classiques : « Ich meine nicht: heraus aus den Theatern und hinein in die AgitpropBewegung, aber ich meine, dass man für die Dramatik auch der Theater sehr viel gewinnen würde aus dem Kampfgeist, der für kleine direkt agitierende Truppen nötig ist und der bei ihnen auch entstehen kann [...]. » Bertolt BRECHT, « Ausführungen vor der Sektion Dramatik zum IV. Deutschen Schriftstellerkongress ", in Große Kommentierte Berliner und Frankfurter Ausgabe, Schriften 3, vol. 23, Frankfurt/Main, Suhrkamp, 1993, p. 368 
performativité la forme théâtrale. Elle devient en ce sens porteuse d'une contre-culture théâtrale qui se développe extra muros, peut créer du dissensus et éveiller la conscience politique du spectateur.

Handke est certainement impressionné par les actions de la Kommune 1 qui n'hésite pas à détourner les événements du réel, à jouer avec la réalité, à la "théâtraliser »- voire à la terroriser - pour mieux rendre visible les rapports de domination qui traversent la société. Cette nouvelle forme de théâtre de rue engagé s'oppose à ce qu'il appelle le «théâtre-théâtre », c'està-dire le théâtre institutionnel, incapable de formuler les besoins de la société dans la mesure où son discours s'est formalisé, où l'héritage brechtien a été " digéré » par l'entreprise théâtrale, devenant un mode de représentation normalisé. Les points de ressemblance établis entre le théâtre brechtien et la Kommune 1 relèvent toutefois d'une construction personnelle de Handke : jamais la Kommune 1 ne s'est référée explicitement à Brecht, ses références théoriques et artistiques étant plutôt à chercher du côté de l'Internationale situationniste, de la performance et du happening américain (et bien sûr des collectifs artistiques comme le Bread and Puppet Theater ou le Living Theater qui, eux, ont pu se référer à Brecht ${ }^{16}$ ). Mais cette analogie entre les actions de la Kommune 1 et le théâtre de Brecht témoigne d'une volonté de renouveler les contenus et les formes du théâtre afin d'en faire une arme (ou au moins un outil dynamique) de la révolution. Il s'agit désormais de concevoir un nouveau type de spectacle qui soit "agissant " et fasse ainsi le lien entre l'agir théâtral et l'agir politique - au risque de semer le doute entre le jeu et la réalité, de brouiller les frontières entre jeu et non-jeu, théâtre et non-théâtre.

Rappelons que Peter Handke, qui n'a que 26 ans à cette époque, bénéficie déjà d'une certaine visibilité dans le champ théâtral ouest-allemand. La création par Claus Peymann de sa pièce Outrage au public (Publikumsbeschimpfung) à Francfort deux ans auparavant (en 1966) a été à la fois un véritable scandale et un grand succès. Dans ce texte d'une forme inhabituelle pour l'époque (une succession de blocs sans dialogue, sans personnage ni action), Handke s'en prend aussi bien aux conventions dramatiques qu'aux attentes du spectateur bourgeois, installé dans un confort esthétique et matériel. En insultant et en outrageant le public, Handke suscite le conflit entre la scène et la salle, afin de remédier à l'épuisement du conflit sur scène, à l'appauvrissement de la forme dramatique. Qu'attendre du théâtre aujourd'hui ? À quoi sert la forme spectaculaire dans la société actuelle ? Ces questions sont toujours présentes quelques années plus tard dans le discours de l'auteur autrichien et berlinois d'adoption. Elles incitent désormais à un mouvement de déterritorialisation, à un bond hors de l'institution: vers un nouveau théâtre de la rue.

16 Le Living Theater a, par exemple, présenté la pièce Antigone en 1967 en suivant la version proposée par Brecht. 


\section{Émergence du théâtre de rue et développement d'une scène off}

On peut analyser quelques exemples de réalisations théâtrales relevant de la subversion de l'espace public, dans l'esprit des formes spectaculaires proposées par la contre-culture étudiante et des performances «agissantes" de la Kommune 1, mais qui dérivent également vers d'autres types de spectacles. Des ateliers de théâtre amateur se sont développés et politisés dans les grandes universités d'Allemagne de l'Ouest tout au long des années 1960 ; de nouveaux groupes se constituent par la suite au contact des mouvements contestataires, en cherchant à associer le travail théâtral à l'action politique dans la tradition de l'agit-prop et en expérimentant de la sorte de nouveaux modes de production. À Berlin-Ouest, on voit ainsi émerger, dans le sillage des manifestations, des spectacles aux formats variés privilégiant l'écriture spontanée et collective.

Das Sozialistische Straßentheater Berlin (West) ou le « Théâtre de Rue Socialiste de Berlin (Ouest) », fondé en 1968, peut être considéré comme un pur produit du mouvement étudiant. C'est l'un des premiers collectifs à revendiquer l'appellation de "théâtre de rue », même si cette dénomination existe depuis les tentatives d'agit-prop de la fin de la République de Weimar ${ }^{17}$. Le 21 avril 1968, ce groupe composé d'amateurs met au point un bref spectacle lors d'une manifestation sur la Wittenberg Platz, qui raconte le putsch militaire survenu un an auparavant en Grèce (ill. 3). Le choix du nom de ce collectif indique clairement la direction dans laquelle le SSB veut inscrire son travail : il s'agit de faire de la forme théâtrale un instrument d'agitation politique dans le combat contre l'impérialisme et pour le socialisme, tout en revendiquant la rue comme espace de jeu libre et ouvert. $\grave{A}$ partir de l'une des rares photographies d'archive de ce spectacle, on peut penser que c'est la parole qui domine cette performance, avec un texte écrit par les participants eux-mêmes à partir de matériaux documentaires. L'expressivité et le sens du jeu ne sont a priori pas des priorités dans ce spectacle où les participants tiennent des poses durant la récitation du texte. Il est intéressant de parcourir le manifeste du collectif, qui constitue une note d'intention pour toutes ses productions. Cette autodéfinition du SSB s'intitule «Critique et autocritique ${ }^{18}:$ on y découvre que la grande peur du groupe demeure la récupération politique ; il s'agit de rester efficace dans la justesse d'un positionnement, sans devenir décoratif, ni être rabaissé à un moment de folklore étudiant.

17 Voir Agnes HÜFNER, Straßentheater, Frankfurt/Main, Suhrkamp, 1970, p. 9. L'ouvrage de Agnes Hüfner est un document essentiel et de première main au sujet de l'émergence du théâtre de rue politisé en Allemagne. 11 présente de nombreux textes et photographies de collectifs des années 1960 et fait également le lien avec l'histoire du théâtre politique, en particulier du théâtre d'agit-prop des années 1920-1930, dont les théories et les pratiques sont exhumées dans les années 1960 et font l'objet d'une relecture attentive.

18 Ibid., p. 285-304. 


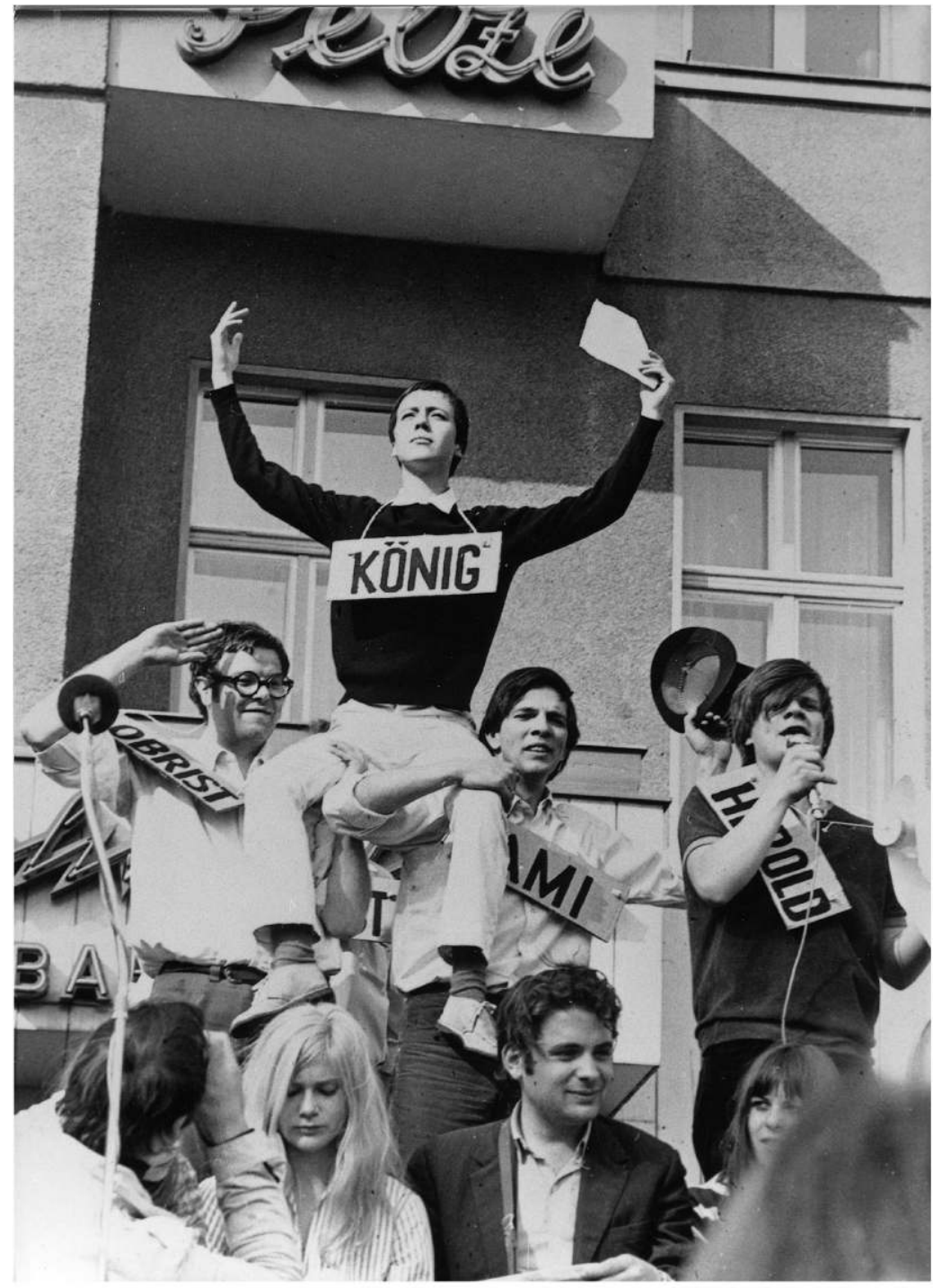

Ill. 3 : Le Théâtre de Rue Socialiste de Berlin-Ouest propose sur la Wittenberg Platz une mise en scène du putsch militaire survenu en Grèce, 21.04.1968. Ullstein Bild, (C) Haertel.

Dans la même veine, la troupe de Théâtre de Rue de Kreuzberg (Kreuzberger Straßentheater) est composée quant à elle uniquement d'étudiants, issus de domaines variés (architecture, cinéma, sociologie), mais pas spécifiquement des études théâtrales, qui écrivent également eux-mêmes leurs textes. Dans un spectacle rudimentaire présenté à la Kottbusser Tor en 1969 Le Confort dans un endroit convenable ou La Rénovation (Komfort in 
günstiger Lage oder Die Sanierung) (ill. 4), il est question des difficultés d'un couple d'ouvriers (« Herr und Frau Schubse »), confronté aux rénovations du quartier de Kreuzberg, et à une possible expulsion de son logement ${ }^{19}$. Ici aussi, le dispositif est très simple : pas de costumes ni de décor pour ce spectacle joué sur le bitume. Une simple banderole entoure les participants, sans toutefois correspondre à un véritable cadre de scène. Il s'agit d'une mise en espace plus que d'un spectacle : dans une attitude parfois nonchalante (une main dans la poche), les participants lisent leur texte, avec de simples pancartes autour du cou pour indiquer le nom de leur personnage. Ceux qui endossent les rôles des profiteurs - Hausbesitzer (« le propriétaire ») Bausenator (« le sénateur des Travaux Publics ») - portent également des masques. De la même manière que pour le SSB, l'élaboration de la forme théâtrale tourne autour de typologies simples. Les personnages représentés sont toujours les représentants d'une classe sociale et s'opposent de manière binaire : l'apprenti contre le directeur, l'ouvrier contre le capitaliste. La note d'intention du collectif informe le lecteur que ce travail a été mené en collaboration avec des sociologues et des ouvriers et qu'il vient soutenir une distribution de tracts. Il s'agit là encore d'un projet amateur, qui vise à informer les passants sur les projets de la mairie pour le quartier et à occuper l'espace public en proposant une agitation essentiellement verbale, sans véritable intention artistique.

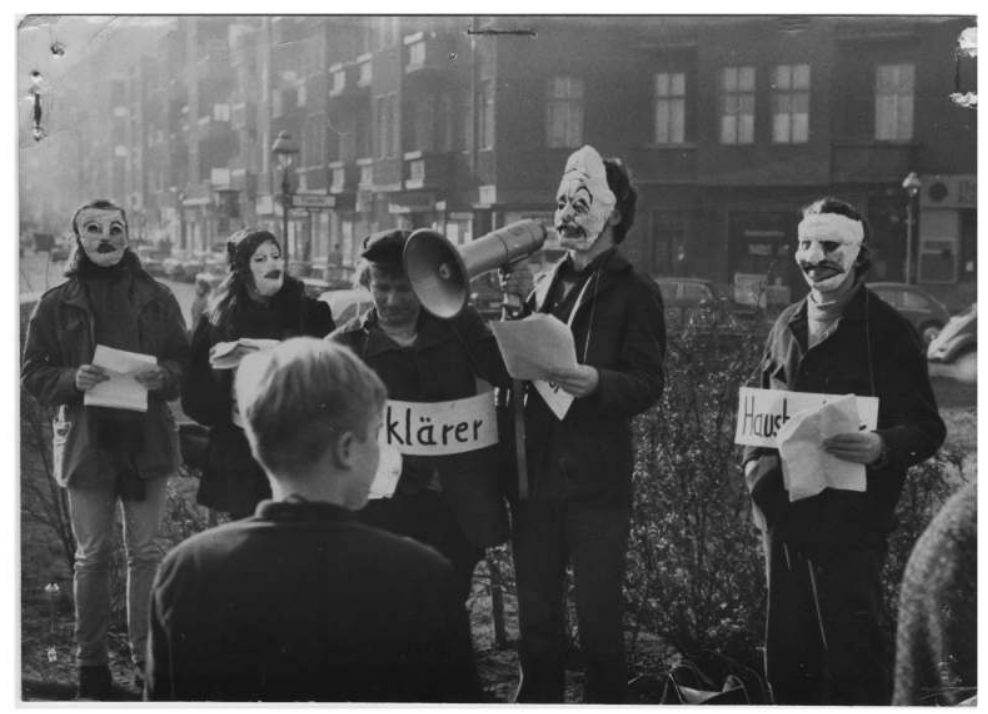

Ill. 4. La troupe du Théâtre de Rue de Kreuzberg présente le spectacle Komfort in günstiger Lage oder Die Sanierung, Kottbusser Tor, juin 1969. D.R.

19 Ce texte très court est également reproduit dans l'ouvrage de A. HÜFNER, ibid., p. 231239. Pour faire le lien avec les années 1920, on peut noter que dans le film de Dudow et Brecht Kuhle Wampe, redécouvert en 1968, la seule brève scène d'agit-prop du film - celle du Portevoix rouge (Das Rote Sprachrohr) - est une scène de résistance à l'expulsion de chômeurs. 
Contrairement à ces deux exemples, certains collectifs de théâtre de rue berlinois ont une véritable ambition artistique. Ainsi, la compagnie Hoffmanns Comic Teater, fondée dès 1963 et politisée sur le tard au contact du mouvement étudiant, est composée de comédiens professionnels qui exploitent la tradition de la Commedia dell'arte. À l'aide de masques et en mettant l'accent sur la corporalité de l'acteur, ce collectif revendique l'appellation de «théâtre de rue » dans un sens ouvert aux innovations, comme une pratique d'expérimentation de tous les langages artistiques (musique, cirque, marionnette et même bande dessinée). Le Hoffmanns Comic Teater propose des séries de numéros courts, choisissant le modèle de la revue, avec une part d'improvisation autour des thèmes choisis. Le public peut également être sollicité, car les acteurs distribuent volontiers des masques aux spectateurs qui sont prêts à participer ou simplement à dialoguer. Le spectacle présenté le $1^{\text {er }}$ mai 1970 à Berlin-Ouest dans le quartier de Reinickendorf (ill. 5) est proposé dans le cadre d'une manifestation contre la fermeture d'un centre culturel alternatif et l'expulsion des jeunes du quartier qui l'animaient - ce qui explique la présence de parents, d'enfants et d'adolescents dans le public ${ }^{20}$. Cette proposition a été élaborée en quatre jours seulement, c'est l'une des spécificités revendiquées par le théâtre de rue, encore émergent à cette époque : jouer sur un mode de production rapide et simple, pour mieux réagir à l'actualité, avec un spectacle démontable et transportable s'adaptant à toutes les situations et aux intempéries. En cas de pluie, le Straßentheater peut aussitôt se replier et se transformer en Kellertheater (« théâtre de sous-sol ») ou Kneipentheater (« théâtre de bistrot ») .

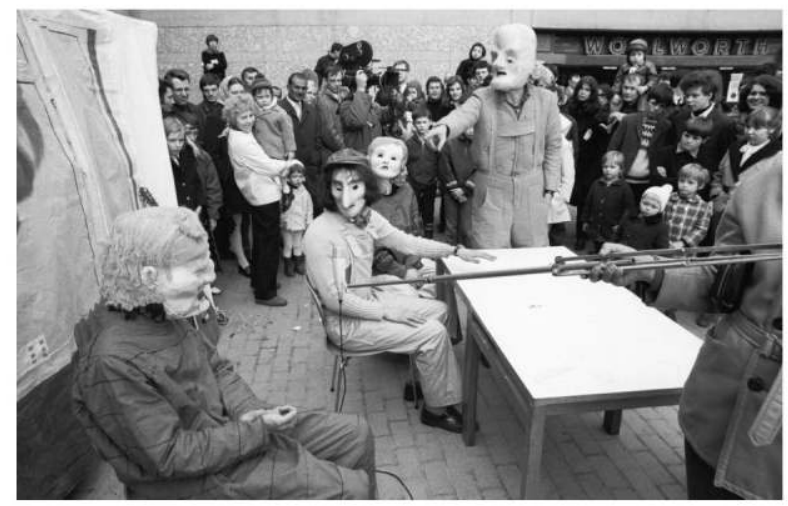

Ill. 5 : Spectacle du Hoffmanns Comic Teater présenté le $1^{\text {er }}$ mai 1970 à Berlin-Ouest. (C) Klaus Mehner.

20 Signalons qu'à la même époque, des cinéastes de la DFFB tournaient un film politique sur les problèmes sociaux des habitants de ce grand ensemble, ce qui montre les interconnexions contre-culturelles du mouvement protestataire de 1968. Cf. l'article d'André Combes dans ce numéro.

21 Ces informations se trouvent dans la note d'intention du Hoffmanns Comic Teater, reproduit dans l'ouvrage d'A. HÜFNER, ibid., p. 250-255. 
Cette scène off émergente apparaît également comme une tentative pour se libérer du système des théâtres publics et de leur hiérarchie. Elle représente le point de départ d'une nouvelle culture théâtrale tournant le dos à l'institution, regroupant amateurs et professionnels et induisant un nouveau rapport au public. Dans ce mouvement contre-culturel du théâtre, il n'existe plus de distinction entre les rôles d'auteur, d'acteur, de dramaturge ou de metteur en scène - ce qui, d'un point de vue professionnel et personnel, est particulièrement attractif : la réalisation de soi et l'expression authentique passent au premier plan, en évitant un parcours laborieux à travers l'institution avant l'obtention de véritables responsabilités pour réaliser ses projets. Ce théâtre indépendant se développe dans les années 1970 et 1980 et devient un mouvement important qu'on appelle par la suite le Freies Theater ( "le théâtre libre ») et qui concerne aujourd'hui encore une partie importante de la scène théâtrale allemande ${ }^{22}$. On compte ainsi, au début des années 1970, une trentaine de " groupes de théâtre libres » en Allemagne de l'Ouest ; après la réunification et seulement pour la ville de Berlin, ce sont plus de 600 groupes divers qui se produisent régulièrement dans la capitale ${ }^{23}$. Sur le plan artistique, ces collectifs ont la particularité d'expérimenter toutes les traditions théâtrales : théâtre pour enfants, théâtre de marionnettes, performance, danse ou cabaret ${ }^{24}$. L'exigence politique ne perdure cependant pas toujours dans la variété des formes et des contenus qui sont proposés par ces groupes indépendants. De plus, pour se maintenir et se développer, la scène théâtrale off (par définition précaire) a besoin d'aides publiques : elle doit s'accommoder des structures existantes et s'adapter aux exigences de la politique culturelle de la ville. Parfois, la liberté acquise par rapport aux grandes scènes institutionnelles se paye par une dépendance envers des fonds privés. Tel est le paradoxe de cette contre-culture théâtrale qui affirme sa liberté et son indépendance : pour exister professionnellement, elle doit sans cesse se repositionner face à l'institution et assumer sa dépendance vis-à-vis des subventions publiques. De la question épineuse de la subversion, on passe à celle de la subvention - pour reprendre les termes du titre de l'ouvrage de Rainer Rochlitz au sujet de l'art contemporain ${ }^{25}$.

22 Pour une présentation détaillée des enjeux et de l'évolution du Freies Theater, je renvoie à l'article de Nikolaus MÜLLER-SCHÖLL, « De la rébellion contre l'institution au théâtre "hors de soi" : le Freies Theater depuis 2000 », in Didier PLASSARD (dir.), Mises en scène d'Allemagne(s), Paris, CNRS Éditions, Les Voies de la création théâtrale, vol. 24, à paraître en 2013.

23 Barbara BÜSCHER, Wirklichkeitstheater, Straßentheater, Freies Theater. Entstehung und Entwicklung freier Gruppen in der Bundesrepublik Deutschland 1968-76, Frankfurt/Main, Bern, Peter Lang Verlag, 1987, p.189-194. Signalons que l'ouvrage de Barbara Büscher, qui propose un certain nombre de données chiffrées, fait également un panorama très détaillé des expérimentations relevant du théâtre de rue dans les années 1970 en RFA.

24 Pour une présentation d'un exemple de renouveau du cabaret berlinois dans les années 1970, consulter l'article de Andreas Häcker sur le groupe de cabarettistes Die 3 Tornados.

25 Rainer ROCHLITZ, Subversion et subvention, Paris, Gallimard, 1994. 


\section{Conclusion}

On voit en définitive de quelle manière les phénomènes contestataires et artistiques autour de 1968, portés par une réflexion théorique importante, mènent à une nouvelle définition de ce que peut et de ce que doit représenter le théâtre dans une nouvelle société. La logique d'opposition à l'ordre établi qui prévaut dans les actions théâtralisées de l'APO et de la Kommune 1 interpelle directement le monde du théâtre, tant du point de vue des formes usitées que des contenus élaborés. Cette nouvelle impulsion qui traverse le champ du théâtre autour des années 1968 peut donc être appelée «contreculture théâtrale » : elle s'exprime d'abord en s'adossant à un mouvement socio-historique contestataire, en radicalisant son rapport à la société (en général) et à l'institution théâtrale (en particulier), notamment dans ce que celle-ci peut avoir de figé, à travers des rapports hiérarchisés et autoritaires. Par la suite, le mouvement contre-culturel qui touche l'activité théâtrale se démultiplie à travers différents types de réappropriation et de formes variées comme le Straßentheater, parfois reprises des expériences contre-culturelles weimariennes. Il connaît également un phénomène d'érosion à travers l'éclatement du Freies Theater - une évolution fréquente de la contre-culture qui a souvent tendance à s'intégrer à la culture dominante.

Berlin-Ouest n'est pas l'unique centre de la contestation en 1968 - celle-ci se manifeste par exemple également dans des villes comme Francfort ou Cologne - et Berlin-Ouest n'est pas non plus la seule ville d'Europe où l'on invente le happening et redécouvre le théâtre de rue. Mais de par sa situation singulière d'enclave, de "ville-frontière", elle se retrouve régulièrement au centre de projets alliant le politique à l'artistique de façon radicale : elle condense d'une certaine façon les expérimentations théâtrales marginales de cette époque et se situe au cœur du balancement des contre-cultures, entre in et off, entre théâtre « libre » et théâtre institutionnel. Par ailleurs, Berlin-Ouest est directement confronté à la vie théâtrale de Berlin-Est de la fin de l'ère autoritaire d'Ulbricht, où le théâtre off est impensable. C'est ainsi que l'on pourrait qualifier le Sonderweg ouest-berlinois : un «chemin à part» qui éclaire le rapport de force entre culture et contre-culture, non pas sous la forme d'une opposition binaire, mais plutôt d'un antagonisme en évolution permanente qui représente la dynamique de la culture elle-même. 